\title{
The effect of signal intensity on comparative judgments of auditory durations'
}

\author{
TRIEVE A. TANNER, JR. AND R. MARK PATTON, NASA, AMES RESEARCH CENTER \\ RICHARD C. ATKINSON, STANFORD UNIVERSITY
}

Human Ss made comparative judgments of the duration of two tones in a forced-choice situation. Pairs of tones with either the same or different intensities were presented with durations from 0.5 to $1.6 \mathrm{sec}$. Comparisons were more accurate when the two tones were of the same intensity than when they were of different intensities, and were most accurate when the tones were of higher intensities. The results are compared with previous findings relating comparative judgments of duration to sensory modality.

In a recent study Tanner et al (1965) investigated comparative judgments of signal duration by varying the modality to which the signals were presented. It was found that in a two-alternative forced-choice situation, human Ss were more accurate with intraauditory comparisons than with either intravisual or intramodal comparisons. Evidence was presented that there was a bias toward judging visual signals to be longer in duration than auditory signals.

The purpose of the study reported here was to determine whether intensity differences between two signals might affect judgments of signal duration in a way comparable to that which has been shown with differences in modality. If the effects of modality and intensity are found to be comparable, the construction of a model of the psychophysics of signal duration should be simplified.

Previous investigations of the effect of intensity on the accuracy of judging auditory durations have yielded conflicting results. Creelman (1962) has discussed several of these studies. His own research supports the hypothesis that intensity is important only to the extent that it contributes to the clear detection of the signal throughout its presentation period.

In the present study Ss made comparative judgments not only of the duration of two tones with the same intensities, as did Creelman's Ss, but also of two tones with different intensities. The latter were included, in part, to determine whether or not a bias existed toward calling either the more or the less intense tone longer. Method

The procedure was similar to that of the previous study by Tanner et al. The Ss were 11 male college students, all of whom were free from gross auditory impairment as determined by an informal interview. None of the Ss had performed the task previously. S sat in a dimly lighted, sound attenuated room. The auditory signals, $1000 \mathrm{cps}$ sinusoids, were presented through earphones (Permoflux, D4S-17) at an intensity of either 86 or $106 \mathrm{db}$, both re 0.002 dyne per $\mathrm{cm}^{2}$. To insure that the "on-times" of the signals did not depend on intensity, the rise and fall were gated instantaneously; therefore audible clicks accompanied the gating. White noise $(100 \mathrm{~K} \mathrm{cps}$, low pass) at $56 \mathrm{db}$ also was presented through the earphones.
Each $S$ performed for a total of 6144 trials, grouped as 12 sessions of 512 trials each. On each trial S's task was to judge which of two successively presented tones was longer by pressing one of two response switches. Three pairings of signal durations were used in the study: 0.5 sec. vs. 0.6 sec., 1.0 sec. vs. 1.1 sec., and $1.5 \mathrm{sec}$. vs, 1.6 sec. Thus the difference in duration of the signals in each of the three pairings $(\Delta t)$ was $0.1 \mathrm{sec}$. As the durations became longer, the relative difference between the incremental duration and that of the briefer signal $(\Delta t / t)$ became smaller. Hereafter the three pairings of signal durations will be designated as $\mathrm{D}(0.5), \mathrm{D}(1.0)$, and $\mathrm{D}(1.5)$, the numeral denoting the duration of the briefer signal of a given pair. Only one of the three pairings was presented during a given session.

Except for the duration of the signals, the intervals within each trial were constant throughout the experiment: the ready period was 1.0 sec., the intersignal interval was $0.8 \mathrm{sec} .$, the response interval was $2.0 \mathrm{sec}$., and the intertrial interval was $2.0 \mathrm{sec}$. During the intertrial interval the background noise was turned off. Its offset identified the end of the response interval, and its onset the start of the next ready period.

On each trial one of four possible pairings of signal intensity was presented: two $106 \mathrm{db}$ tones $\left(\mathrm{S}_{\mathrm{HH}}\right)$, two $86 \mathrm{db}$ tones $\left(\mathrm{S}_{\mathrm{LL}}\right.$ ), a $106 \mathrm{db}$ tone followed by an $86 \mathrm{db}$ tone $\left(\mathrm{S}_{\mathrm{HL}}\right)$, or an $86 \mathrm{db}$ tone followed by a $106 \mathrm{db}$ tone $\left(\mathrm{S}_{\mathbf{L H}}\right)$.

The eight trial types, consisting of four pairings of intensities and two orders of presenting the duration pairings (first or second signal longer), were presented in a new randomized order during each experimental session.

\section{Results and Discussion}

Table 1 presents both the proportion of correct responses, $\operatorname{Pr}(\mathrm{C})$, made by each $\mathrm{S}$ with each of the 12 combinations of duration and intensity pairings, and the mean values of $\operatorname{Pr}(C)$ for the 11 Ss. Figure 1 presents the mean value of $\operatorname{Pr}(C)$ plotted against $D(t)$, with a separate curve for each of the intensity pairings. As would be expected, $\operatorname{Pr}(C)$ is a decreasing function of $D(t)$.

The hypothesis that the accuracy of comparative time judgments is not related to intensity pairing is rejected $(p<0.001$; Friedman Two-Way Analysis of

\begin{tabular}{|c|cccc|cccc|cccc|}
\hline \multirow{2}{*}{ SUBJECT } & \multicolumn{4}{|c|}{$0(0.5)$} & \multicolumn{4}{c|}{$\mathrm{D}(1.0)$} & \multicolumn{4}{c|}{$\mathrm{D}(1.5)$} \\
\cline { 2 - 7 } & $S_{H H}$ & $S_{L L}$ & $S_{H L}$ & $S_{L H}$ & $S_{H H}$ & $S_{L L}$ & $S_{H L}$ & $S_{L H}$ & $S_{H H}$ & $S_{L L}$ & $S_{H L}$ & $S_{L H}$ \\
\hline 1 & .99 & .97 & .85 & .65 & .87 & .86 & .66 & .55 & .65 & .72 & .68 & .50 \\
2 & .91 & .90 & .90 & .94 & .86 & .87 & .85 & .82 & .78 & .78 & .78 & .78 \\
3 & .95 & .94 & .93 & .95 & .79 & .81 & .80 & .82 & .78 & .77 & .79 & .78 \\
4 & .93 & .84 & .74 & .91 & .85 & .76 & .68 & .83 & .74 & .74 & .66 & .75 \\
5 & .88 & .79 & .76 & .68 & .76 & .73 & .69 & .66 & .66 & .62 & .66 & .61 \\
6 & .77 & .70 & .66 & .74 & .64 & .61 & .67 & .67 & .62 & .56 & .59 & .64 \\
7 & .96 & .92 & .91 & .91 & .80 & .81 & .75 & .79 & .72 & .72 & .67 & .73 \\
8 & .63 & .62 & .63 & .64 & .60 & .57 & .56 & .58 & .58 & .57 & .53 & .54 \\
9 & .96 & .93 & .90 & .87 & .80 & .75 & .75 & .82 & .66 & .65 & .68 & .67 \\
10 & .73 & .70 & .65 & .69 & .66 & .64 & .62 & .60 & .60 & .59 & .59 & .56 \\
11 & .92 & .92 & .86 & .91 & .82 & .79 & .75 & .73 & .70 & .68 & .64 & .66 \\
\hline MEAN & .88 & .84 & .80 & .81 & .77 & .75 & .71 & .72 & .68 & .67 & .66 & .66 \\
\hline
\end{tabular}

Table 1. Pr(C) for $S_{H H}, S_{L L}, S_{H L}, S_{L H}$, under the three duration pairings for individual $S s$, and averaged for the $11 \mathrm{Ss}$. 


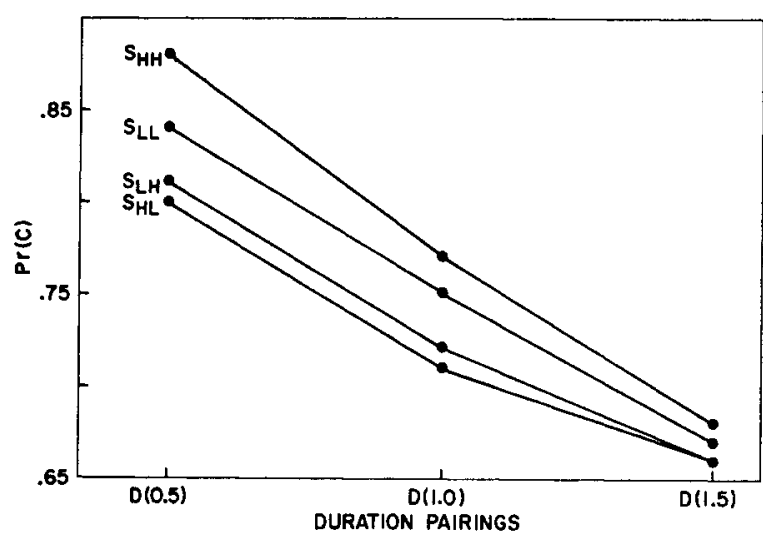

Fig. 1. Mean probability of a correct response.

Variance; Siegel, 1956). As shown in Fig. 1, the $S_{H H}$ comparisons were the most accurate. This superior accuracy with $S_{\mathrm{HH}}$ does not support Creelman's conclusion that the intensity of a tone affects the accuracy of judging its duration only so far as detecting the tone is involved. Both the $106 \mathrm{db}$ and the $86 \mathrm{db}$ tones were clearly detectable in the background noise of $56 \mathrm{db}$. Creelman's conclusion was based on Ss' comparisons of signals of the same intensity only. When only $\mathrm{S}_{\mathrm{HH}}$ and $S_{L L}$ are considered in the present experiment, the hypothesis that accuracy of performance is unrelated to intensity pairing is again rejected $(p=0.02$; Sign Test; Siegel, 1956). Comparisons of the duration of tones of the same intensity $\left(\mathrm{S}_{\mathrm{HH}}\right.$ and $\mathrm{S}_{\mathrm{LL}}$ ) were more accurate than the comparisons of tones with different intensities $\left(S_{H L}\right.$ and $\left.S_{L H}\right),(p=0.033$; Sign Test).

On the trials with different intensities ( $\mathrm{S}_{\mathrm{HL}}$ and $\mathrm{S}_{\mathbf{L H}}$ ) the overall probability of judging the $86 \mathrm{db}$ tone to be longer than the $106 \mathrm{db}$ tone was 0.48 . This suggests an absence of bias toward judging either of the two intensities as longer, since each was in fact longer on half of these trials. That this unbiased behavior was typical is suggested by the fact that the probability of calling the $86 \mathrm{db}$ tone longer was between 0.44 and 0.56 for 8 of the 11 Ss.

When the results reported here are considered in relation to those obtained with different modalities, several conclusions are suggested. Comparative judgments of duration are more accurate when the two signals being compared are alike than when they are different, either in modality or, in the case of tones, in intensity. Also, changes of either the tone intensity or the modality influence accuracy, such that the durations of tones of higher intensity and the durations of tones rather than lights are compared more accurately. Whereas it was reported previously that S's judgments were biased toward calling lights longer in duration than tones, no such bias was found regarding the two tone intensities.

Certain aspects of the data reported here can be given a fairly simple theoretical interpretation. Assume that the onset and offset of the tone marking a temporal interval determine the "on-period" of an internal clock that behaves as a Poisson counter. For the two-alternative, forced-choice task assume that on each trial separate counts are made for the first and the second temporal intervals. The values of these two counts are denoted as $c_{1}$ and $c_{2} . S$ then compares the two counts against a criterion $\mathbf{x}$ and responds according to the following rule:

Select interval $\left\{\frac{1}{2}\right\}$ as longer if $c_{1}-c_{2}$ is $\{\leqslant\}$. For $x=0, S$ judges the first interval to be longer, if $c_{1}>c_{2}$; whereas if $c_{1} \leqslant c_{2}$ he judges the second interval longer.

Assume that the rate at which a counter is driven is a function of the intensity of the tone; the more intense the tone, the faster the counter. From this model the probability of a correct response, conditional on the various intensity pairings, is predicted to be:

$\operatorname{Pr}\left(\mathrm{C} \mid \mathrm{S}_{\mathrm{HH}}\right)>\operatorname{Pr}\left(\mathrm{C} \mid \mathrm{S}_{\mathrm{LL}}\right)>\operatorname{Pr}\left(\mathrm{C} \mid \mathrm{S}_{\mathrm{HL}}\right)=\operatorname{Pr}\left(\mathrm{C} \mid \mathrm{S}_{\mathrm{LH}}\right)$ Furthermore, the model predicts that all of these probabilities will decrease, approaching 0.50 as $D(t)$ increases and $\Delta \mathrm{t}$ remains constant. A similar account may be given for the results of the cross-modality study cited previously, if it is assumed that auditory signals induce a faster counting rate than visual signals.

Although the model provides a good account of the simpler aspects of these data, some of the more detailed features require a more complex formulation. For example, the model, as it has been presented, assumes that the counting rate is independent of $D(t)$. However, if the counting rate associated with either the high or the low intensity tone is estimated from the data of the present experiment, the rate per unit time tends to decrease as $D(t)$ increases. Furthermore, as $D(t)$ increases, it appears that the counting rates for both high and low intensity tones approach a common asymptote. A more detailed theoretical evaluation of these data and the results of research currently being conducted will be given in a later paper.

\section{References}

Creelman, C. D. Human discrimination of auditory duration. $J$. Acoust. Soc. Amer., 1962, 34, 582-593.

Siegel, S. Nonparametric Statistics. New York: McGraw Hill, 1965.

Tanner, T. A., Patton, R. M., \& Atkinson, R. C. Intermodality judgments of signal duration. Psychon. Sci., 1965, 2, 271-272.

Note

1. The authors wish to express their appreciation to Julie Sadenwater for her assistance in collecting the data. 